\title{
Establishment and characterization of human non-small cell lung cancer cell lines
}

\author{
JIANGCHAO LI ${ }^{1}$, HONG YANG ${ }^{2}$, LEILEI CHEN ${ }^{3}$, YAN LI ${ }^{1}$, YINGHUI ZHU ${ }^{1}$, YONGDONG DAI ${ }^{1}$, \\ KAI CHEN $^{1}$, JIAOYU AI ${ }^{1}$, TINGTING ZENG ${ }^{1}$, XUEYING MAO ${ }^{1}$, \\ LULU LIU ${ }^{1}$, XIAODONG LI $I^{2}$ and XIN-YUAN GUAN ${ }^{1,3}$ \\ ${ }^{1}$ State Key Laboratory of Oncology in Southern China, and ${ }^{2}$ Department of Thoracic Surgery, \\ Sun Yat-Sen University Cancer Center, Guangzhou; ${ }^{3}$ Department of Clinical Oncology, \\ The University of Hong Kong, Pokfulam, Hong Kong SAR, P.R. China
}

Received July 18,2011; Accepted September 28, 2011

DOI: $10.3892 / \mathrm{mmr} .2011 .613$

\begin{abstract}
Non-small cell lung cancer (NSCLC), a highly malignant tumor, is common in China and is associated with a very poor 5 -year survival rate. To better understand the cancer biology of this disease, we report here the establishment of three new NSCLC cell lines, SCC210011, SCC211441 and ACC212102, from the tumor tissue of three NSCLC patients. By histological analysis, we found that all three cell lines displayed the typical features of endothelial cancer cells. The population doubling times of SCC210011, SCC211441 and ACC 212102 cells were 42, 38 and 25 h, respectively. Our cytogenetic studies indicated that these cell lines exhibit structural and numerical chromosomal abnormalities. Furthermore, the tumorigenicity in nude mice was confirmed, and H\&E staining results revealed that they resembled the primary tissue. These newly established cell lines may serve as useful models for studying the molecular pathogenesis of NSCLC.
\end{abstract}

\section{Introduction}

Lung cancer is the most common primary tumor and the most common cause of cancer-related mortality in males worldwide $(1,2)$. Lung cancer is mainly classified as non-small cell lung cancer (NSCLC) or small cell lung cancer (SCLC). NSCLC, which is the most malignant tumor, is common in China and is associated with a very poor 5-year survival rate (3). However, little is known regarding the carcinogenesis and metastasis

Correspondence to: Professor Xin-Yuan Guan, State Key Laboratory of Oncology in Southern China, Sun Yat-Sen University Cancer Center, Room 605, 651 E. Dongfeng Road, Guangzhou 510060, P.R. China E-mail: xyguan@hkucc.hku.hk

Dr Leilei Chen, Department of Clinical Oncology, The University of Hong Kong, Room L10-56, Laboratory Block, 21 Sassoon Road, Pokfulam, Hong Kong SAR, P.R. China

E-mail: pollyllc@hku.hk

Key words: non-small cell lung cancer, cell line, lung cancer of this tumor. Although several lung cancer cell lines have been established, their hereditary capacity has been lost due to countless passages. Therefore, to provide a deeper understanding of tumorigenesis, the cancer microenvironment, metastasis (4), diagnosis and drug resistance, it is necessary to establish additional lung cancer cell lines. To date, there are a limited number of lung cancer cell lines in China (5-8). As a result, we established and characterized Chinese human lung cancer cell lines that we named SCC210011, SCC211441 and ACC212102. These cell lines are quite useful for studying lung cancer pathology and treatment.

\section{Materials and methods}

Clinical specimens. Resection specimens were obtained from a 74-year-old Chinese male, a 55-year-old Chinese male and a 74-year-old Chinese male who underwent surgical resection at the Cancer Center, Sun Yat-Sen University (Guangzhou, China). The clinical features of the 3 NSCLC patients are outlined in Table I. The patients were admitted to the Department of Thoracic Surgery of the Cancer Center, Sun Yat-Sen University. All patients had signed informed consents on the use of clinical specimens for medical research. Samples used in this study were approved by the Committee for Ethical Review of Research at Sun Yat-Sen University.

Primary culture and cell line establishment. All tumors were verified by pathology, and the specimens from patients were cultured in Dulbecco's modified Eagle's medium (DMEM) with $10 \%$ fetal bovine serum (FBS) plus penicillin and streptomycin. The size of each tumor specimen was $\sim 1 \mathrm{~cm}^{3}$. No lymph node or distant metastasis was detected at the time of resection. The three tumor tissues (210011, 211441 and 212102) were histopathologically classified as moderately to poorly differentiated squamous carcinoma, squamous carcinoma and adenocarcinoma, respectively, according to the H\&E staining results. The tissue specimens were washed twice with phosphate-buffered saline (PBS), cut into 0.5-2 $\mathrm{mm}^{3}$ pieces, again washed with $\mathrm{PBS}$, centrifuged at $1,000 \mathrm{rpm}$ for $5 \mathrm{~min}$ and finally rinsed twice in serum-free 
Table I. Non-small cell lung cancer patient characteristics.

\begin{tabular}{lccccc}
\hline Patient no. & Age & Gender & Prior-therapy & Tumor site & Cell line designation \\
\hline 210011 & 74 & Male & None & Left lower lobe & SCC210011 \\
211441 & 55 & Male & None & Left lower lobe & SCC211441 \\
212102 & 70 & Male & None & Right lower lobe & ACC212102 \\
\hline
\end{tabular}

medium. The pellet was suspended in complete DMEM with $20 \%$ FBS, penicillin $(100 \mathrm{U} / \mathrm{ml})$ and streptomycin $(100 \mu \mathrm{g} /$ ml) (Invitrogen, San Diego, CA, USA), and seeded into plastic flasks, which were overturned to keep the plaster pieces on the flasks. Following 3-4 h, the flasks were turned over to submerge the sections in the medium. The flasks were incubated at $37^{\circ} \mathrm{C}$ for 2-3 weeks, following which the FBS was reduced to $10 \%$ in the media. The primary cells were then subcultured when they reached $70-80 \%$ confluence. During the period of continuous subculture propagation, the cells were frozen with freezing medium containing 70\% DMEM, $10 \%$ FBS and $10 \%$ dimethyl sulfoxide (DMSO), and stored in liquid nitrogen. Following thawing, cells were able to be propagated in culture without obvious changes in the growth rate and morphology. The three cell lines were designated SCC210011, SCC211441 and ACC202102, with each number representing the patient hospitalization record.

Growth characteristics in vitro. Passages 35, 31 and 30 were studied for SCC210011, SCC211441 and ACC202102, respectively, to estimate the population doubling time. A suspension of $1 \times 10^{3}$ cells was plated in each well of a 96-well plate, and the growth rate of cells was examined using a cell proliferation kit (Roche, Mannheim, Germany) and microplate reader for 6 days . The growth curve was plotted and the doubling time of each cell line was estimated during the logarithmic growth phase.

Anchorage-independent growth assay in soft agar. A bottom layer of $0.6 \%$ agarose with $10 \%$ FBS in $35-\mathrm{mm}$ plastic dishes was prepared, and single-cell suspensions of each cell line in $0.3 \%$ agarose with DMEM and $10 \%$ FBS were placed on top. For each plate, $1 \times 10^{3}$ cells were tested, and the colonies were scored after 2-3 weeks. Colony-forming efficiency was determined as the number of colonies per number of tumor cells, multiplied by 100 (data not shown).

Tumorigenicity in nude mice and $H \& E$ staining analysis. All protocols were performed following approval of the Institutional Animal Care and Use Committee of Sun Yat-Sen University. The cells were subcutaneously injected into one of the flanks of 3- to 4-week-old nude male mice. The animals were observed for the development of tumors each week and sacrificed 4-6 weeks following injection. The tumor tissues were fixed with $10 \%$ formalin and a routine histopathological examination was performed by H\&E staining and compared to the primary patient tissue.

Cytogenetic analysis. Chromosome harvest was performed following short-term culture. Metaphase chromosomes were stained by standard trypsin-G banding method. In total, 72-85 metaphases were characterized. The description of karyotypic abnormalities followed the recommendations of the International System for Human Cytogenetic Nomenclature (9).

\section{Results}

Morphology and culture characteristics. SCC210011, SCC211441 and ACC212102 cells were successfully isolated from lung cancer tissues (Fig. 1A) and formed sparse colonies within 52, 42 and 38 days of primary culture, respectively (Fig. 1B, upper panel). Once cancer cell growth commenced, it tended to proceed progressively with minimal cell death. The contaminating fibroblasts gradually decreased until finally disappearing (Fig. 1B, lower panel). The cells began to grow quickly around the seventh to tenth passage and were propagated for more than 30 generations thereafter. The cultured SCC210011, SCC211441 and ACC212102 cells grew as adherent monolayers with characteristic epithelial morphologic features (Fig. 1B, lower panel). The cells maintained morphology that was consistent with the primary culture throughout the following 30 passages and more. Following thawing, the cryopreserved cells were able to be propagated in culture without a noticeable change in growth and morphology. The growth curves of the three cell lines are shown in Fig. 2A. The population doubling time of SCC210011, SCC211441 and ACC212102 cells was approximately 42, 38 and $25 \mathrm{~h}$, respectively. These three cell lines were able to produce recognizable colonies in soft agar (data not shown).

Tumorigenicity in nude mice. These three cell lines exhibited strong tumorigenic capabilities in athymic nude mice. Within 4-6 weeks following subcutaneous injection of SCC210011, SCC211441 and ACC212102 cells, tumor formation was visible at the site of inoculation in 40,75 and $75 \%$ of mice, respectively (Fig. 2B and C). By histological analysis, the xenograft tumor nodules displayed moderate to poor differentiation (Fig. 2D), which was similar to the description of the corresponding original lung tumors (Fig. 1A).

Cytogenetic analysis. G-banding analysis demonstrated that SCC210011, SCC211441 and ACC212102 cells were all triploid. Three representative karyotypes are shown in Fig. 3, and the median number of chromosomes found in SCC210011, SCC211441 and ACC212102 cell lines were 65, 58 and 62, respectively. By cytogenetic analyses, we confirmed that these three cell lines were human in origin and had numerous structural and numerical chromosome abnormalities (Fig. 3). 
A
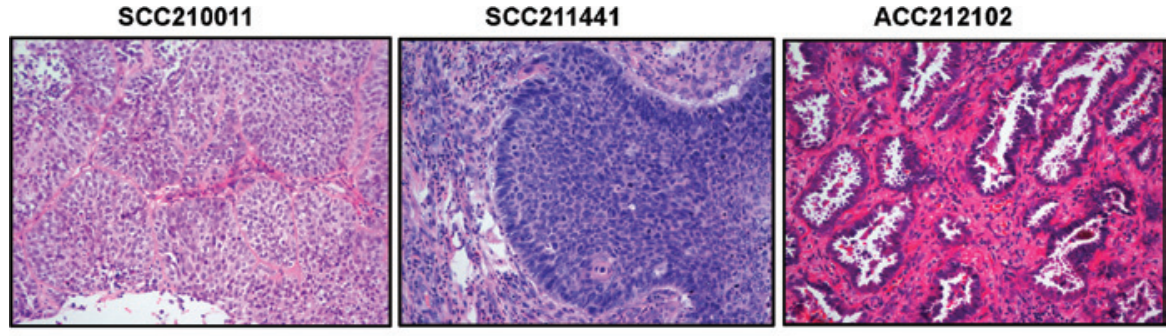

B
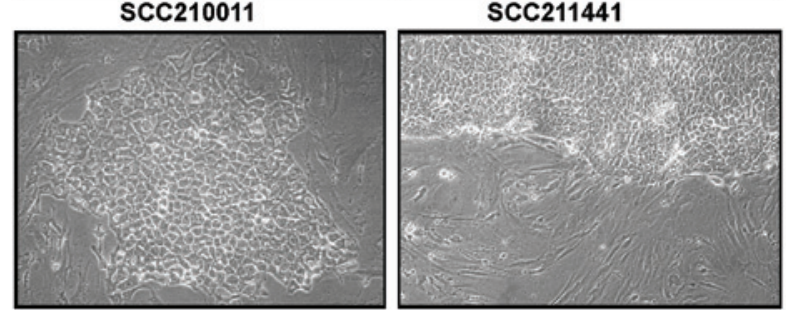

ACC212102

$\operatorname{scc210011}$

$\operatorname{ScC211441}$
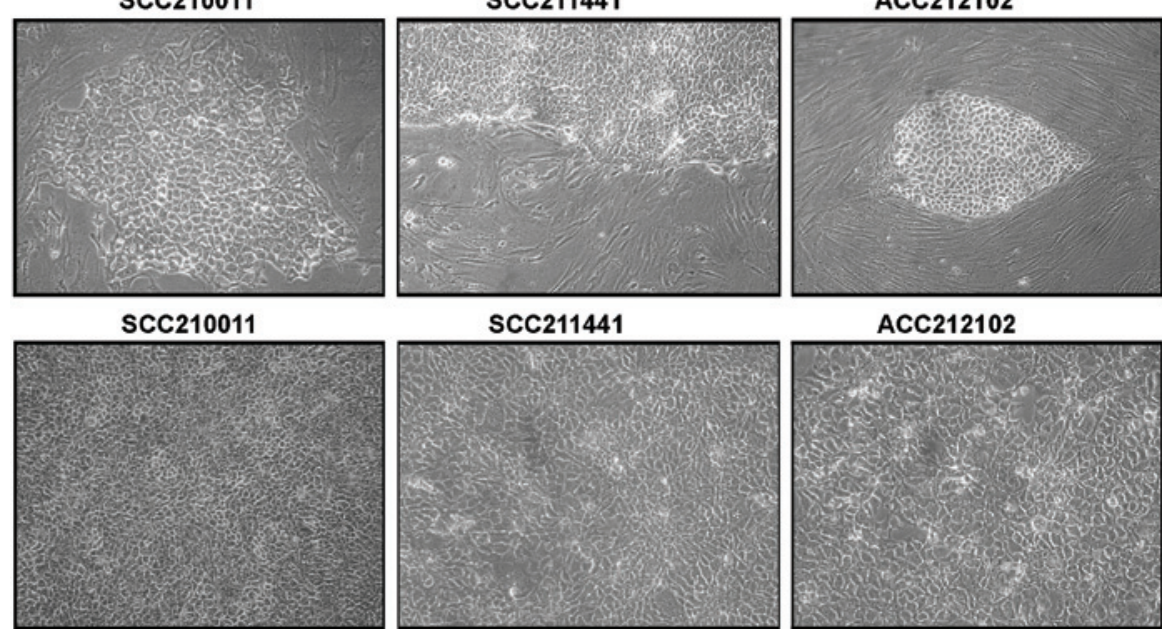

ACC212102

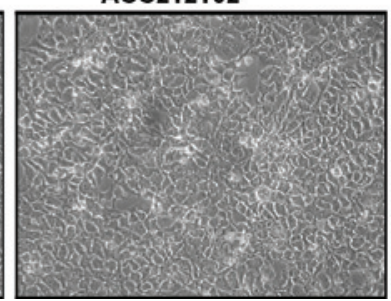

Figure 1. (A) Histology of three human non-small cell lung cancer tissues: SCC210011, squamous carcinoma; SCC211441, squamous carcinoma; ACC212102, adenocarcinoma. These tumors show typical malignant epithelia in primary tumor tissue (magnification, x200). (B) SCC210011, SCC211441 and ACC212102 cells in short-term culture. These cells exhibit typical malignant epithelia in morphology and monolayer sheets (upper panels; magnification, x100). Cells exhibit typical malignant epithelia in morphology when passaged for 25, 20 and 19 times, respectively (lower panels; magnification, x100).

A
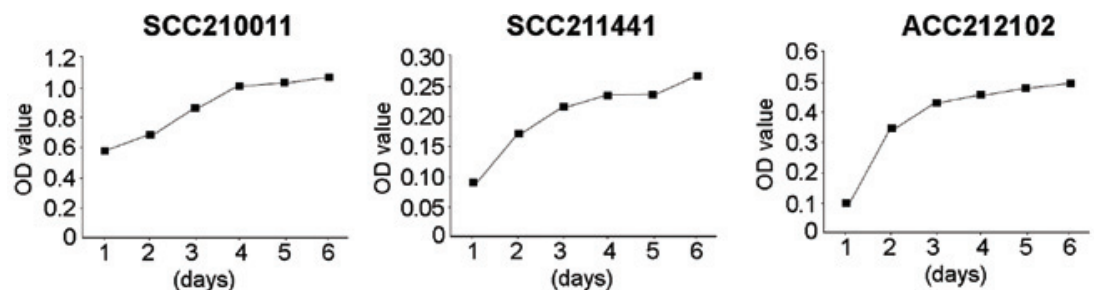

B

Properties of three lung cancer cell lines

\begin{tabular}{ccccc}
\hline Cell line & Tumorigenic/Percentage* & Histology of tumors & Doubling time (hr) & Chromosome number \\
\hline SCC210011 & Yes $/ 40 \%$ & Squamous carcinoma & 42 & 65 \\
SCC211441 & Yes $/ 75 \%$ & Squamous carcinoma & 38 & 58 \\
ACC212102 & Yes $/ 75 \%$ & Adenocarcinoma & 25 & 62 \\
\hline
\end{tabular}

"Total $2 \times 10^{6}$ of cells have been injected subcutaneously into nude mice.

C

$\operatorname{scc} 210011$

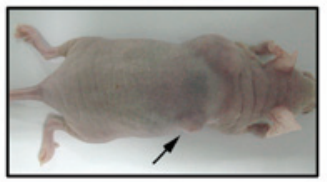

$\operatorname{scc210011}$

D

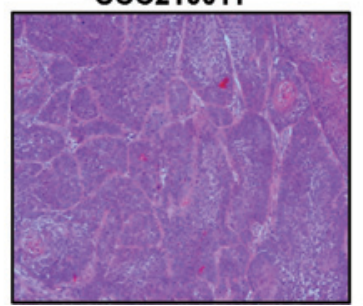

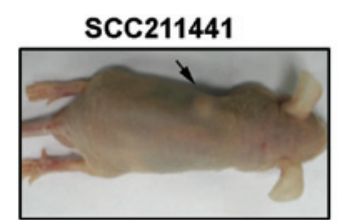

$\operatorname{scc211441}$

ACC212102

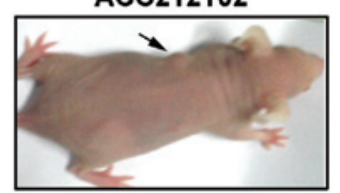

ACC212102

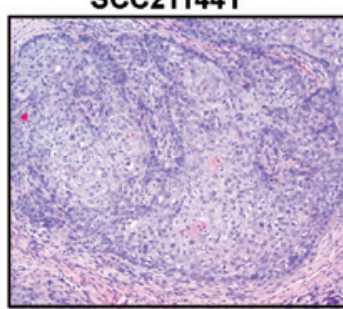

Figure 2. (A) Growth curves of SCC210011, SCC211441 and ACC212102 cell lines. (B) Properties and tumorigenic abilities of three non-small cell lung cancer cell lines. (C) Cells $\left(2 \times 10^{6}\right)$ (SCC210011, SCC211441 or ACC212102) were subcutaneously injected into nude mice. Arrows indicate tumor formation following 4-6 weeks of injection. (D) Representative H\&E staining of harvested xenograft tumors revealed the primary tumor phenotypes and poor differentiation (magnification, x100). 
$\operatorname{SCC210011~}$

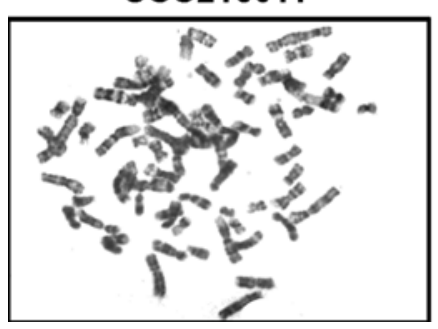

SCC211441

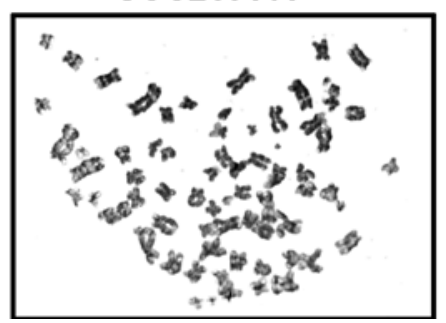

ACC212102

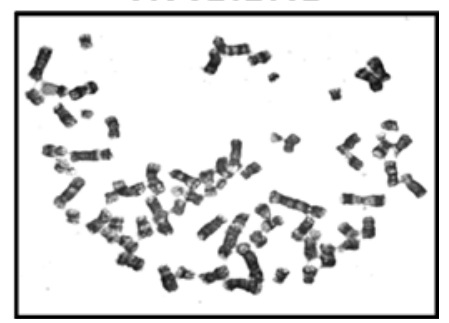

Figure 3. Representative G-banded karyotypes of SC210011, SC211441 and ACC212102 cell lines.

\section{Discussion}

In general, surgical tissues have been used to establish human lung cancer cell lines, and various functional lung cancer cell lines have been reported (10-12). Nonetheless, the incidence of lung cancer remains globally high. Thus, employment of new cell lines to study the mechanism of lung carcinoma, particularly for NSCLC is needed. In the present study, three NSCLC cell lines were established from Chinese patients. These established pure cell lines may be used for tumor progression and cancer environmental studies that cannot normally be performed using human tissue specimens.

We named these cell lines SCC210011, SCC211441 and ACC212102. The three cell lines grew as adherent monolayers with characteristic epithelial morphology and their population doubling time was approximately 42,38 and $25 \mathrm{~h}$, respectively. The three cell lines were continuously grown for 3-4 months, while undergoing more than 30 passages; in addition, cell growth continued even following recovery from cryopreservation. The cell lines also exhibited an ability to form colonies in soft agar. Furthermore, the three cell lines were subcutaneously injected into nude mice and resulted in tumors. We found that the histological features of the tumor resembled those of the original primary tumor and maintained a consistent morphology as compared to the primary culture by $H \& E$ staining. Furthermore, the cell lines were analyzed by cytogenetic analysis, and we confirmed that these cells were of human origin, that they were tumor cells and that chromosomal aberrations were involved, as confirmed by the chromosome G-banding method. To date, only a limited number of studies have investigated the cytogenetic changes in lung cancer and our understanding of the molecular carcinogenesis of the disease remains limited. Our findings reveal that these cell lines are triploid. They display various chromosomal alterations when compared to other lung cell lines that have been previously characterized in our laboratory (7). Our results suggest that the chromosomal abnormalities detected in these newly established cell lines may play a crucial role in lung cancer carcinogenesis.

At present, the available established NSCLC cell lines have been propagated for long periods. Therefore, certain biological characteristics may have been altered and/or lost and, thus, they may not be appropriate for the study of co-culture and the cancer microenvironment. For this reason, primary tissue cultures may provide a valuable in vitro model with which to study the various aspects of tumor biology, cellular and molecular carcinogenesis, as well as biomarkers for early diagnosis and drug response to new therapeutic agents, all of which are necessary for a better understanding of lung cancer. In this regard, there is a great need to further update the collection of lung cancer cell lines with a new supply. We expect that the application of new cell lines may greatly accelerate human lung cancer cell studies.

\section{Acknowledgements}

This study was supported in part by grants from the National Natural Science Foundation of China (30772475, 30700820 and 30971606), the Sun Yat-Sen University 'Hundred Talents Program' (85000-3171311), and the Nation Key Sci-Tech Special Project of China (2008ZX10002-022).

\section{References}

1. Ferlay J, Shin HR, Bray F, Forman D, Mathers C and Parkin DM: Estimates of worldwide burden of cancer in 2008: Globocan 2008. Int J Cancer 127: 2893-2917, 2010.

2. Kamangar F, Dores GM and Anderson WF: Patterns of cancer incidence, mortality, and prevalence across five continents: defining priorities to reduce cancer disparities in different geographic regions of the world. J Clin Oncol 24: 2137-2150, 2006.

3. Parkin DM, Bray F, Ferlay J and Pisani P: Global cancer statistics, 2002. CA Cancer J Clin 55: 74-108, 2005.

4. Yu L, Hui-chen F, Chen Y, et al: Differential expression of RAB5A in human lung adenocarcinoma cells with different metastasis potential. Clin Exp Metastasis 17: 213-219, 1999.

5. Yan FC, Wang QQ, Ruan YH, Ma LJ, Jia JT and Jin KW: Establishment and biological characteristics of lung cancer cell line XWLC-05. Ai Zheng 26: 21-25, 2007.

6. Yang S, Su J, Shi M, et al: establishment and characterization of a novel Chinese human lung adenocarcinoma cell line CPA-Yang2 in immunodeficient mice. Zhongguo Fei Ai Za Zhi 12: 1055-1060, 2009.

7. Tai AL, Fang Y, Sham JS, et al: Establishment and characterization of a human non-small cell lung cancer cell line. Oncol Rep 13: 1029-1032, 2005.

8. Lam DC, Girard L, Suen WS, et al: Establishment and expression profiling of new lung cancer cell lines from Chinese smokers and lifetime never-smokers. J Thorac Oncol 1: 932-942, 2006.

9. Knutsen T, Bixenman HA, Lawce $\mathrm{H}$ and Martin PK: Chromosome analysis guidelines preliminary report. Cancer Genet Cytogenet 52: 11-17, 1991.

10. Kume M, Taguchi T, Okada H, et al: Establishment and molecular cytogenetic characterization of non-small cell lung cancer cell line KU-T1 by multicolor fluorescence in situ hybridization, comparative genomic hybridization, and chromosome microdissection. Cancer Genet Cytogenet 179: 93-101, 2007.

11. Sharifzadeh S, Owji SM, Pezeshki AM, et al: Establishment and characterization of a human large cell lung cancer cell line with neuroendocrine differentiation. Pathol Oncol Res 10: 225-230, 2004.

12. Harada R, Uemura Y, Kobayashi M, et al: Establishment and characterization of a new lung cancer cell line (MI-4) producing high levels of granulocyte colony stimulating factor. Jpn J Cancer Res 93: 667-676, 2002. 